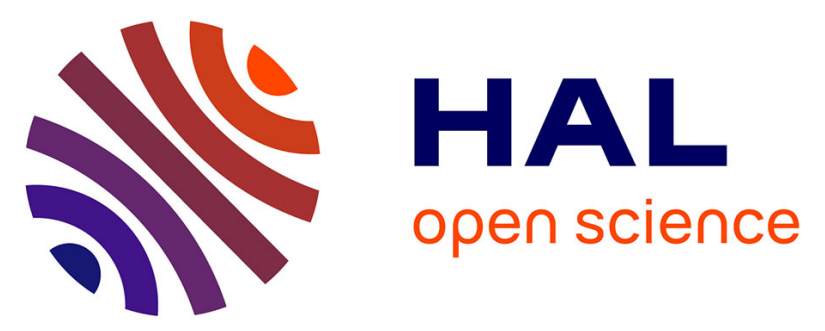

\title{
Tuning Local Nanoparticle Arrangements in TiO2-Polymer Nanocomposites by Grafting of Phosphonic Acids
}

\author{
Anne-Caroline Genix, Celine Schmitt-Pauly, Johan G. Alauzun, Thomas \\ Bizien, P. Hubert Mutin, Julian Oberdisse
}

\section{To cite this version:}

Anne-Caroline Genix, Celine Schmitt-Pauly, Johan G. Alauzun, Thomas Bizien, P. Hubert Mutin, et al.. Tuning Local Nanoparticle Arrangements in TiO2-Polymer Nanocomposites by Grafting of Phosphonic Acids. Macromolecules, 2017, 50 (19), pp.7721-7729. 10.1021/acs.macromol.7b01371. hal-01627751

\section{HAL Id: hal-01627751 \\ https://hal.science/hal-01627751}

Submitted on 19 Nov 2018

HAL is a multi-disciplinary open access archive for the deposit and dissemination of scientific research documents, whether they are published or not. The documents may come from teaching and research institutions in France or abroad, or from public or private research centers.
L'archive ouverte pluridisciplinaire HAL, est destinée au dépôt et à la diffusion de documents scientifiques de niveau recherche, publiés ou non, émanant des établissements d'enseignement et de recherche français ou étrangers, des laboratoires publics ou privés. 


\title{
Tuning local nanoparticle arrangements in $\mathrm{TiO}_{2}$-polymer nanocomposites by grafting of phosphonic acids
}

\author{
Anne-Caroline Genix ${ }^{1 *}$, Céline Schmitt-Pauly ${ }^{1,2}$, Johan G. Alauzun ${ }^{2}$, Thomas Bizien ${ }^{3}$, P. Hubert \\ Mutin $^{2}$, Julian Oberdisse ${ }^{1}$ \\ ${ }^{1}$ Laboratoire Charles Coulomb (L2C), UMR 5221 CNRS, Université de Montpellier, F-34095 Montpellier, \\ France \\ ${ }^{2}$ Institut Charles Gerhardt Montpellier, UMR 5253 CNRS-UM-ENSCM, Université de Montpellier, F-34095 \\ Montpellier Cedex 5, France \\ ${ }^{3}$ SOLEIL Synchrotron, L'Orme des Merisiers, Gif-Sur-Yvette, 91192 Saint-Aubin, France \\ * Author for correspondence: anne-caroline.genix@umontpellier.fr
}




\begin{abstract}
The influence of surface-modification of $\mathrm{TiO}_{2}$-nanoparticles with phosphonic acid molecules on the structure of polymer nanocomposites has been studied by small-angle scattering and transmission electron microscopy. The grafting of phosphonic acids was done by phase transfer into chloroform, and polymer nanocomposites have been formulated by solvent casting with two polymers of slightly different hydrophobicity, PMMA and PEMA. By analyzing the shape of the scattering curves around the interparticle correlation peak, and in particular the depth of the correlation hole, information on nearest neighbor correlations between nanoparticles is obtained. While local nanoparticle arrangements are found to be independent of the global particle volume fraction, they are controlled by the degree of hydrophobicity of the alkyl phosphonic acid grafts with respect to hydrophobicity of matrix. Quantitative analysis of the correlation hole thus evidences the fine-tuning of local nanocomposite structure with phosphonic acids.
\end{abstract}




\section{Introduction}

Polymer nanocomposites (PNCs) formulated by incorporation of usually inorganic nanoparticles (NPs) in polymer matrices possess optical, mechanical, or dynamical properties, ${ }^{1-3}$ which depend to a great extent on the structure or dispersion of the NPs in the polymer. ${ }^{4,5}$ The latter ultimately determines transport properties ${ }^{6,7}$ or reinforcement ${ }^{8-10}$, i.e., transport of momentum across the sample. The average local arrangement of NPs depends on their nature, on the properties of the interface, and on the formulation path: individual and well-defined model NPs dispersed by some physico-chemical means ${ }^{11-13}$, e.g., will form different structures than, say, agglomerates of very polydisperse primary nanoparticles of industrial origin usually incorporated by strong mechanical mixing ${ }^{14,15}$.

Moreover, the properties of the interface may be tuned by evolving from, e.g., bare oxides $\left(\mathrm{SiO}_{2}, \mathrm{TiO}_{2}\right.$, $\mathrm{Al}_{2} \mathrm{O}_{3} \ldots$ ) with surface $\mathrm{OH}$ groups interacting with the polymer matrix to oxides grafted with oligo- or polymers. Surface modifications with macromolecules have been shown to have a strong influence on the final dispersion state, both in industrial ${ }^{16,17}$ and model systems ${ }^{18,}{ }^{19}$. On the other hand, surface modifications with small molecules, usually based on silane chemistry, are ubiquitous in industrial applications ${ }^{20-22}$ as well as in model systems ${ }^{23-25}$. Recently, we have grafted hydrophilic and hydrophobic alkyl phosphonic acids (PA) on alumina-silica NPs, and studied the impact of surface chemistry on NP interactions in aqueous suspension by scattering techniques ${ }^{26}$. The grafting reaction was shown to have a strong influence on colloidal stability, which was obviously related to the hydrophobicity of the grafts. We then investigated the formation and structure of PNCs made with these grafted NPs in a poly(ethyl acrylate) polymer matrix using a latex-route and solvent casting from water ${ }^{27}$. A combination of transmission electron microscopy (TEM) image analysis and small-angle scattering allowed the estimation of the local particle volume fraction $к$ in aggregates, as a function of the global particle concentration. It was found that at low concentrations $(\Phi<5 \% \mathrm{v}), \kappa$ stayed approximately constant, around $10 \%$. At higher concentration, aggregates became denser, independently of the type of graft, with the notable exception of the bare particles, which formed the densest aggregates with $\mathrm{\kappa} \approx 28 \%$, much higher than $\approx 17 \%$ obtained with the most hydrophobic graft. This was interpreted as the effect of a dense hydrophobic layer impeding direct contact between particle surfaces, and improving compatibility with the hydrophobic polymer matrix. ${ }^{27}$

The aim of this article is to present the structural analysis of polymer nanocomposites with surface modified nanoparticles using alkyl phosphonic acids of different hydrophobicity. The latter is determined by the length of the aliphatic chain, $\mathrm{C}_{8}, \mathrm{C}_{12}$ or $\mathrm{C}_{18}$. A completely different grafting pathway than the one just mentioned ${ }^{27}$ was explored, following recently reported successful solvent transfer from water into chloroform and other solvents by grafting of PAs. ${ }^{28}$ In this previous study, the initial dispersion state in aqueous suspension presented small (equivalent sphere radius ca. $32 \mathrm{~nm}$ ) and tenuous (internal volume fraction $\approx 11 \%$ ) aggregates made of primary nanoparticles. Upon phase 
transfer into chloroform with $\mathrm{C}_{8} \mathrm{PA}$ and $\mathrm{C}_{12} \mathrm{PA}$ grafts, these aggregates remain unchanged, whereas they grow slightly with the longest graft, $\mathrm{C}_{18} \mathrm{PA}$. Changing $\mathrm{pH}$ or ionic strength in aqueous suspension induced aggregation in this medium due to the reduction of electrostatic repulsion, but was reversible during phase transfer, and thus did not modify the aggregation state in chloroform, presumably due to the formation of a protective layer. Moreover, it was shown for the $\mathrm{C}_{18} \mathrm{PA}$ grafts that upon heating the aggregates in chloroform could be broken down to their initial size in water, which implies that aggregation is reversible. ${ }^{28}$ It results for the present article that such aggregates formed in suspension may be reorganized in the polymer matrix, under the decisive influence of the polymer-graft interactions. Furthermore, the tenuous initial aggregates found in solution may interpenetrate during film formation, losing their shape. The primary particle is thus the building block appropriate for describing all aggregates in nanocomposites structures.

In this work, PNCs have been formulated by solvent casting from chloroform. TEM and small-angle X-ray scattering (SAXS) have then been used to characterize the proximity of nanoparticles inside the polymer matrix, expressed by the compacity к, i.e., the local density of NPs in aggregates. We have recently proposed a straightforward analysis of $\kappa$ applicable to any assembly of polydisperse nanoparticles ${ }^{14,27,29}$. It is based on the average interactions between neighboring NPs which result in a well-defined signature - a correlation hole in q-space - close to the peak associated with particles in contact $(\mathrm{q} \approx \pi / \mathrm{R})$. The polydispersity-dependent parameters of a mapping procedure of the correlation hole onto local density have been determined, allowing the analysis of experimental scattering data without further numerical work. In the present article, this procedure is used to study the impact of surface modification with phosphonic acids on the local density of $\mathrm{TiO}_{2}$-nanoparticle assemblies in PNCs.

\section{Materials and methods}

Nanoparticles and surface modification: $\mathrm{TiO}_{2} \mathrm{NPs}$ (LS101) were a gift from Lotus Synthesis in the form of a stable aqueous suspension (95\% anatase, $5 \%$ brookite), at $\mathrm{pH}<1$, with $23 \%$ w solid content. Zetametry of bare particles in water at $\mathrm{pH} 2$ gave $50 \pm 11 \mathrm{mV}$, indicating a positive surface charge. We have measured an average diameter of $5.2 \mathrm{~nm}$ over more than one hundred NPs by TEM, and smallangle scattering data of the diluted suspension in water (also shown in Figure 1) was well described by a log-normal distribution function $\left(\mathrm{R}_{0}=2.4 \mathrm{~nm}, \sigma=0.3\right)$, corresponding to an average radius of 2.5 $\mathrm{nm}$, in good agreement with the TEM analysis. ${ }^{28}$ For the determination of the NP volume fraction, a density of $3.43 \mathrm{~g} / \mathrm{cm}^{3}$ was measured by picnometry, and the SAXS scattering length density was 2.80 $10^{11} \mathrm{~cm}^{-2}$. On a larger scale, aggregation of the primary NPs into tenuous $\mathrm{TiO}_{2}$ clusters was observed (see Figure 1a), with a radius corresponding to an equivalent homogeneous sphere of $32 \mathrm{~nm} .{ }^{28}$ 
The phosphonic acids (PAs) used for surface modification were $n$-octyl, $n$-dodecyl, and $n$-octadecyl phosphonic acid, labeled $\mathrm{C}_{8} \mathrm{PA}, \mathrm{C}_{12} \mathrm{PA}$, and $\mathrm{C}_{18} \mathrm{PA}$, respectively, of molar mass 194, 250, and 334 $\mathrm{g} / \mathrm{mol}$. All of the PAs were purchased from SiKÉMIA. Surface modification of $\mathrm{TiO}_{2} \mathrm{NPs}$ with PAs $(1.7 \mathrm{mmol} / \mathrm{g})$ by phase transfer was performed by mixing the aqueous sol diluted to $1 \% \mathrm{w}$ of $\mathrm{TiO}_{2}$ with chloroform containing the PAs, as described in ref ${ }^{28}$. The nominal grafting density given by the number of phosphorus atoms per $\mathrm{nm}^{2}$ of NP surface determined by BET $\left(200 \mathrm{~m}^{2} / \mathrm{g}\right)$ is $5 \mathrm{P} / \mathrm{nm}^{2}$, which corresponds to a dense monolayer. Given the tenuous nature of the $\mathrm{TiO}_{2}$ clusters, it is supposed that grafting takes place around all primary NPs.

Polymer matrices and nanocomposite formulation: Nanocomposites have been made with poly(methyl methacrylate) $\left(\mathrm{PMMA}, \mathrm{M}_{\mathrm{w}}=95 \mathrm{~kg} \cdot \mathrm{mol}^{-1}\right.$, polydispersity index PI $=1.8$ by size exclusion chromatography, density $1.17 \mathrm{~g} / \mathrm{cm}^{3}$, scattering length density $1.0910^{11} \mathrm{~cm}^{-2}, \mathrm{~T}_{\mathrm{g}}=113^{\circ} \mathrm{C}$ by DSC at $20 \mathrm{~K} / \mathrm{min}$ ) and poly(ethyl methacrylate) $\left(\mathrm{PEMA}, \mathrm{M}_{\mathrm{w}}=350 \mathrm{~kg} \cdot \mathrm{mol}^{-1}, \mathrm{PI}=2.3\right.$, density $1.15 \mathrm{~g} / \mathrm{cm}^{3}$, scattering length density $1.0710^{11} \mathrm{~cm}^{-2}, \mathrm{~T}_{\mathrm{g}}=66^{\circ} \mathrm{C}$ by DSC at $20 \mathrm{~K} / \mathrm{min}$ ) from Scientific Polymer. Nanocomposites were formulated by mixing a polymer solution $(10 \% \mathrm{w})$ with a suspension of PAmodified NPs $(0.7 \%$ w), both in chloroform, without further purification. Mixtures were dried about $12 \mathrm{~h}$ at $50^{\circ} \mathrm{C}$ to obtain nanocomposite films of approximate thickness $0.6 \mathrm{~mm}$, and $\mathrm{NP}$ volume fractions $\Phi$ ranging from 0.5 to $10 \% \mathrm{v}$. The impact of annealing at $140^{\circ} \mathrm{C}$ for $48 \mathrm{~h}$ was studied with samples of NPs modified with a combination of phosphonic acid and $n$-hexylamine (HA, 2.5 molecules per $\mathrm{nm}^{2}$ added to chloroform in addition to the PAs) in order to reduce aggregation (see refs ${ }^{30,31}$ for details). HA grafts to $\mathrm{Ti}^{4+}$ sites and therefore does not compete with PAs for $\mathrm{TiOH}$ surface groups. ${ }^{30,31}$ Thermogravimetric analysis (TGA) was performed under air (50 ml/min, $\left.20 \mathrm{~K} / \mathrm{min}\right)$, and the mass loss between 200 and $600^{\circ} \mathrm{C}$ was used to determine filler mass fractions of nanocomposites, and thus volume fractions using the densities given above. Due to scattering of the TGA data around the nominal volume fractions caused by sample heterogeneities, only the nominal fractions, termed $\Phi$, are reported. Note that PMMA-nanocomposites with bare NPs have been formulated for comparison, using a completely different procedure as PAs - only HA is also insufficient - are necessary for phase transfer: dry bare NP powders have been dispersed by ultrasonication in chloroform before mixing with the polymer solution, followed by drying.

Structural analysis: Small-angle X-ray scattering was performed on instrument Swing at Synchrotron Soleil in Saint Aubin (France) at a wavelength $\lambda=1.38 \AA(9 \mathrm{keV})$ with two different sample to detector distances $\mathrm{D}=1.1 \mathrm{~m}$ and $6.5 \mathrm{~m}$, yielding a total q-range from $1.210^{-3}$ to $0.7 \AA^{-1}$. Primary data reduction using Foxtrot, matrix subtraction, and superposition at large q were systematically applied. TEM pictures were recorded with a JEOL 1200 EXII apparatus at $100 \mathrm{kV}$. Samples embedded in resin were cut in slices of nominal thickness $\mathrm{d}=70 \mathrm{~nm}$. Image analysis was performed with Gwyddion and Image J. 
Data analysis of small-angle scattering: The experimental (also called 'apparent') structure factor S(q) describing the spatial arrangement of polydisperse primary NPs is obtained by dividing the intensity I(q) scattered by nanocomposites by the average primary NP form factor $\mathrm{P}_{0}(\mathrm{q})$. For monodisperse particles, a straightforward development of the Percus-Yevick (PY) equation at low q ${ }^{32-}$ ${ }^{34}$, or equivalently the Carnahan-Starling equation ${ }^{35}$, gives the following limit with $\alpha$ set to one, $\kappa$ representing the volume fraction:

$$
S_{P Y}(q \rightarrow 0)=\frac{(1-\alpha \kappa)^{4}}{(1+2 \alpha \kappa)^{2}}
$$

In presence of NP polydispersity, a single structure factor is not sufficient to describe the thermodynamics of the system. In eq.(1), we have introduced the parameter $\alpha$ to allow a generalization to polydisperse spheres ${ }^{14}$, based on numerical simulations. Partial structure factors are added up to calculate the experimental structure factor; they have been used to describe the concentration- and polydispersity-dependence of the low-q limit of the structure factor of a simulated assembly of polydisperse hard spheres. ${ }^{29}$ Values for $\alpha$ as a function of polydispersity have been given in this reference. Eq.(1) for $\mathrm{S}(\mathrm{q} \rightarrow 0)$ has been used to describe the experimentally observed structure factor, and in particular the correlation hole in q-space $\mathrm{S}_{0}$. This depression of the structure factor results from local NP interactions and is related to the NP density in aggregates, or equivalently the local volume fraction, termed compacity, к. For the $\mathrm{TiO}_{2}$-NPs studied here of polydispersity $30 \%, \alpha=0.59$ is used 29. The procedure is thus to deduce the experimental $S_{0}$ from I(q) and $P_{0}(q)$, and use eq.(1) to estimate the local NP compacity к.

\section{Local density of $\mathrm{TiO}_{2}$-assemblies in PMMA-nanocomposites}

The structure of PMMA polymer nanocomposites with bare $\mathrm{TiO}_{2}$ nanoparticle and volume fractions ranging from 0.5 to $10 \% \mathrm{v}$ has been studied by SAXS. Note that these samples could not be produced by phase transfer due to the absence of PAs (see section II), and can thus not be compared directly to PA-containing samples. It is nonetheless instructive to study their properties. In Figure 1a, the reduced small-angle intensities for a nanocomposite series with bare NPs are shown, and compared to the particle form factor measured in dilute suspension and rescaled to the same contrast and concentration. This particle form factor displays two levels of structure as outlined in the materials section: a high-q regime corresponding to the primary particles, well described by a small size and large polydispersity (average radius $2.5 \mathrm{~nm}, 30 \%$ polydispersity, deduced from the average primary nanoparticle form factor $\mathrm{P}_{0}(\mathrm{q})$ obtained as high-q fit in Figure 1a), and a superstructure representing a small cluster of these primary particles of about $32 \mathrm{~nm}$ radius. From the low-q intensity of the cluster form factor with 
respect to the level of $\mathrm{P}_{0}(\mathrm{q})$, one can estimate the average number of primary particles per cluster (ca. 30 ), which together with the radius of $32 \mathrm{~nm}$ shows that clusters are very tenuous. During film formation, these objects may rearrange and interpenetrate: only the primary beads remain unchanged. These beads are thus the natural building units of the nanocomposite structure, which is why $P_{0}(q)$ is used in our quantitative analysis. This approach is validated by the modification of the intermediate scattering (correlation hole, indicating densification, also Figure 1a) after nanocomposite formation.

The intensity curves of the nanocomposites are superimposed to the particle form factor at the highest q-values only. In the Guinier region of the primary particles $\left(\mathrm{q} \approx 0.05 \AA^{-1}\right)$, the scattered intensity is well below the form factor, forming what we term the correlation hole in q-space. At low q, finally, the scattered intensity increases strongly, and does not level off, thus not giving any indication on finite-size large-scale structure. Moreover, the differences in the low-q upturns cannot be clearly assigned to the nanoparticle structure, as voids or crazes may alter the scattering here. It is therefore not possible in the framework of the present study to conclude on the control of large-scale structures by local interactions. One may note that the steepest increase seems to be correlated with the lowest correlation hole, which is a consequence of the scattering invariant ${ }^{36}$. The depth of the correlation hole, however, does not depend strongly on $\Phi$, indicating that the local densities are mostly independent of the global particle concentration. A quantitative analysis of the local volume fractions based on eq.(1) will be presented below. Finally, it is worth mentioning that several identical samples have been produced independently, finding virtually perfect reproducibility of the SAXS data.
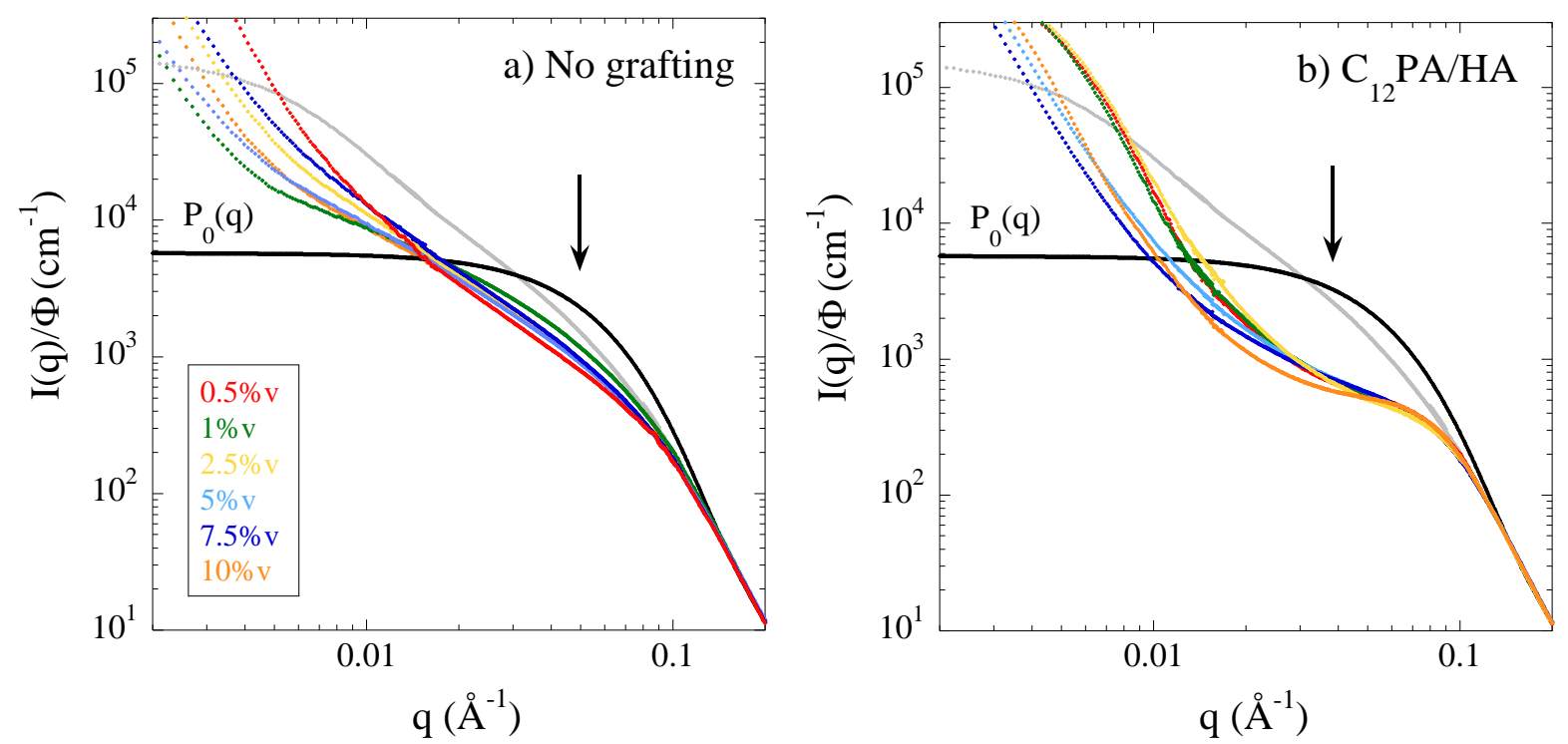

Figure 1: Reduced intensity $\mathrm{I}(\mathrm{q}) / \Phi$ of annealed $\mathrm{TiO}_{2}-\mathrm{PMMA}$ nanocomposites for nominal volume fractions $\Phi$ up to $10 \% \mathrm{v}$ as indicated in the legend. (a) Without grafting, (b) with $\mathrm{C}_{12} \mathrm{PA} / \mathrm{HA}$. The grey curve is the measured NP form factor, and the black one the one of the primary bead $\mathrm{P}_{0}(\mathrm{q})$ identified with the smaller structural level. The arrow indicates the position of the correlation hole. 
Three different grafts, $\mathrm{C}_{8} \mathrm{PA}, \mathrm{C}_{12} \mathrm{PA}$, and $\mathrm{C}_{18} \mathrm{PA}$, have been used for the surface modification of the $\mathrm{TiO}_{2}$-NPs prior to nanocomposite formulation. In Figure 1b, the effect of $\mathrm{C}_{12} \mathrm{PA}$-grafting is shown for an analogous series in $\Phi$, in the presence of hexylamine (HA) (see SI for $\mathrm{C}_{8}$-PA and $\mathrm{C}_{18}-\mathrm{PA}$ ). The scattered intensities display the same shape as the ones of the bare particle-PNCs, with a concentration-independent correlation hole. The depth of the correlation hole, however, is more pronounced, corresponding to a higher local density, to be quantified below. This comparison illustrates the impact of PA, but one must keep in mind that these PNCs are necessarily formulated in a different way in absence of PA, which also influences the final dispersion. We therefore compare PNCs with different surface modification but identical formulation, i.e., undergoing phase transfer.

The influence of the different grafts on the final PMMA-nanocomposite structure is shown in Figure $2 \mathrm{a}$, for $\Phi=5 \% \mathrm{v}$, again in comparison with the primary bead form factor $\mathrm{P}_{0}(\mathrm{q})$ in order to highlight the importance of the correlation hole around $\mathrm{q} \approx 0.05 \AA^{-1}$. The division of $\mathrm{I}(\mathrm{q})$ by $\mathrm{P}_{0}(\mathrm{q})$ gives the experimental structure factor, and the corresponding functions are shown in Figure 2b. As a first result visible in this representation, the terminology of a correlation hole in q-space becomes now obvious, as indeed $\mathrm{S}(\mathrm{q})$ presents a pronounced dip in the q-range preceding the NP interaction peak located at $\pi / \mathrm{R} \approx 0.125 \AA^{-1}$. The hole is positioned at $\mathrm{q}^{*}$ in the graph, and the height of the structure factor there, $\mathrm{S}_{0}$, ranges from 0.15 to 0.25 . The hole is much deeper than one would expect from the average concentration of $5 \% \mathrm{v}$ using eq.(1), which would be around $\mathrm{S}_{0}=0.7$. Particles are thus locally clustered together in much denser zones. A second piece of information on the clusters can be deduced from the general shape of the scattering curves in Figures 1 and 2. The size of the clusters must be larger than 150 to $200 \mathrm{~nm}$ for most samples, because otherwise the low-q intensity or structure factor would display a cross-over towards the corresponding Guinier region; one may note that such a feature seems to exists for the $\mathrm{C}_{18} \mathrm{PA}$, suggesting the presence of smaller clusters. Next, the different grafts are found to affect the scattering around $\mathrm{q}^{*}$, and thus the local density. The correlation hole is deepest for $\mathrm{C}_{8} \mathrm{PA}$, and then becomes shallower for $\mathrm{C}_{12} \mathrm{PA}$ and $\mathrm{C}_{18} \mathrm{PA}$, indicative of a lower local density. For the last sample, one may note that the shape of the interaction peak is different, and its position shifted to the left. This is probably a consequence of the $\mathrm{C}_{18} \mathrm{PA}$ layer, which not only affects the center-to-center distances between NPs, but might also contribute slightly to the form factor scattering. A consequence of the latter point would be that the shape of $\mathrm{S}(\mathrm{q})$ obtained by division could be modified artificially in the corresponding q-range, as observed. Finally, in absence of grafting, a considerably lower local density is found. As we will see shortly, it is still higher than the nominal (global) volume fraction, indicating that there is still clustering, but of lower density. As already mentioned, these samples with bare NPs cannot be compared directly to the grafted ones, due to the different formulation procedure (no phase transfer), but it is interesting to see that the correlation hole reflects the different spatial arrangements of NPs. Complementary data for the structure with different grafts at higher NP volume fractions are shown in the SI, and summarized in the quantitative analysis in section V. 

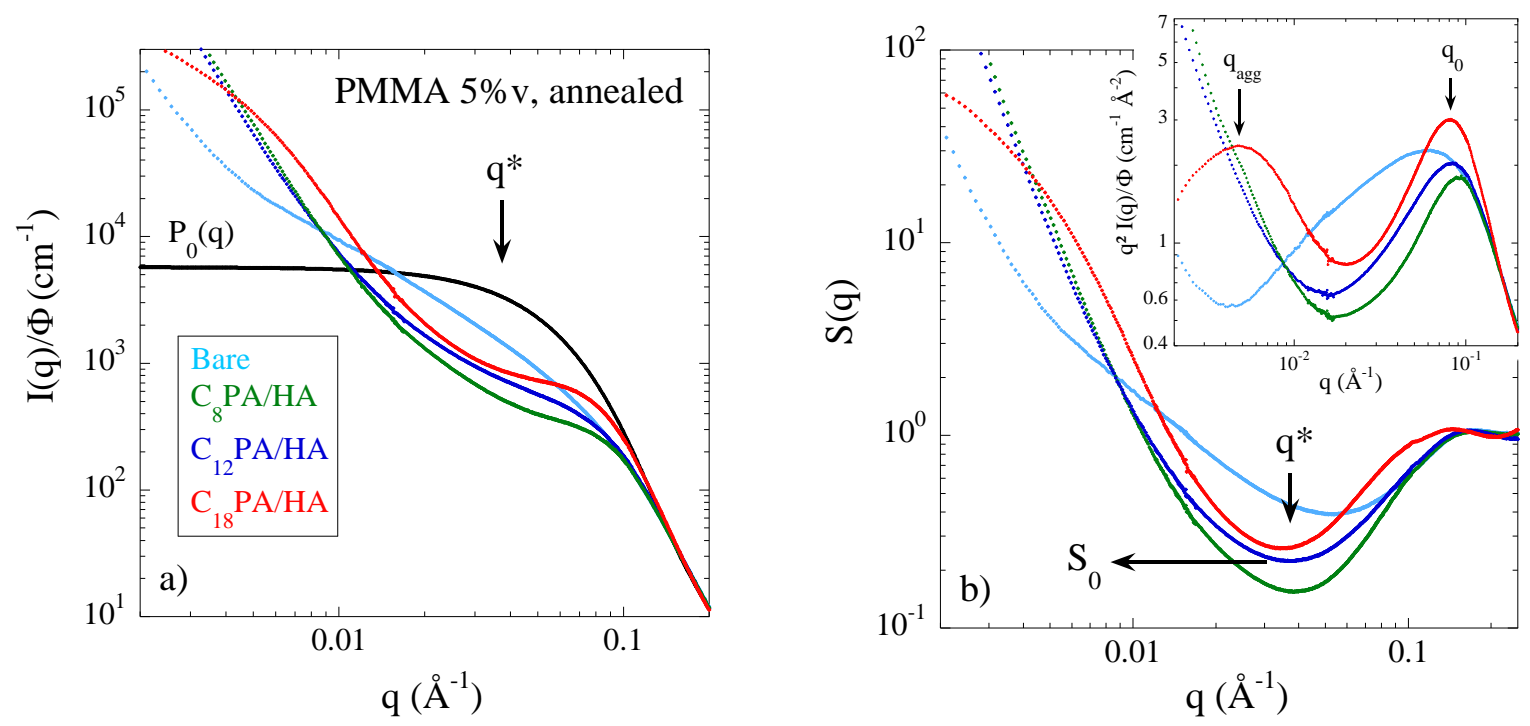

Figure 2: a) Reduced intensity $\mathrm{I}(\mathrm{q}) / \Phi$ of annealed $\mathrm{TiO}_{2}-\mathrm{PMMA}$ nanocomposites at nominal volume fraction $\Phi=$ $5 \% \mathrm{v}$, in absence of grafting, and with $\mathrm{C}_{8} \mathrm{PA} / \mathrm{HA}, \mathrm{C}_{12} \mathrm{PA} / \mathrm{HA}$, and $\mathrm{C}_{18} \mathrm{PA} / \mathrm{HA}$, respectively. b) Structure factor $\mathrm{S}(\mathrm{q})=\mathrm{I}(\mathrm{q}) / \mathrm{P}_{0}(\mathrm{q})$ of the same samples. Kratky representations are shown in the inset.

In the inset of Figure $2 b$, the Kratky presentation $-q^{2} I(q)$ vs $q-$ is shown in order to illustrate the sensitivity of the scattering between the correlation hole $\left(\mathrm{q}^{*}\right)$ and the interaction peak to grafting. The peak moves to the left with grafting, which corresponds to greater center-to-center distances of a nanometer, i.e. typically a molecular size, and is attributed to the effect of the grafted layer. Moreover, the $\mathrm{C}_{18} \mathrm{PA}$-sample presents a second maximum at lower angles, at $0.005 \AA^{-1}$, which highlights the lowq curvature of the same sample visible in Figure 2a. It corresponds to a size of some $125 \mathrm{~nm}$, which may represent a cluster size in these samples.

The series of 5\%-nanocomposites with different grafts has also been studied by TEM and is shown in Figure 3, for two different magnifications. Note that we have checked large-scale homogeneity with pictures taken in different zones (see SI). Nanocomposites made with bare NPs - without phase transfer - are shown in the top row. They form very large assemblies on the micron-scale, suggesting phase separation into pure polymer zones, and those containing nanoparticles. On the right-hand-side, a zoom of a nanoparticle-containing zone is shown. Surprisingly, it does not look as dense in NPs as one might have expected, i.e. polymer is present around the particles. The second and third line in Figure 3 correspond to pictures of PNCs with the lowest mass grafts, $\mathrm{C}_{8} \mathrm{PA}$, resp. $\mathrm{C}_{12} \mathrm{PA}$, both with HA. The large-scale structures shown on the left have been broken up into well-separated clusters of approximate size $200 \mathrm{~nm}$, a magnified view of which is shown on the right. The first effect of the graft is thus to improve the dispersion, and reduce the nanoparticle containing zones into clusters of smaller size. This is interpreted as the result of a better compatibilization of the NPs with the polymer matrix 
after surface modification. With $\mathrm{C}_{18} \mathrm{PA}$ and $\mathrm{HA}$, finally, the structures are shown in the bottom row of Figure 3. They are even smaller and more evenly distributed in the matrix. The approximate size range now approaches $100 \mathrm{~nm}$, thus confirming the tendency of the SAXS result shown in Figure 2, where the $\mathrm{C}_{18} \mathrm{PA}$-intensity was seen to show a curvature at the lowest angles, resulting in a low-q peak in the Kratky presentation (corresponding to $125 \mathrm{~nm}$ ). An equivalent series of TEM pictures for the 2.5\%samples for all grafts is shown in the SI. It presents the same structural features as discussed here, indicating that the global NP volume fraction is not a critical parameter as already seen by SAXS above.
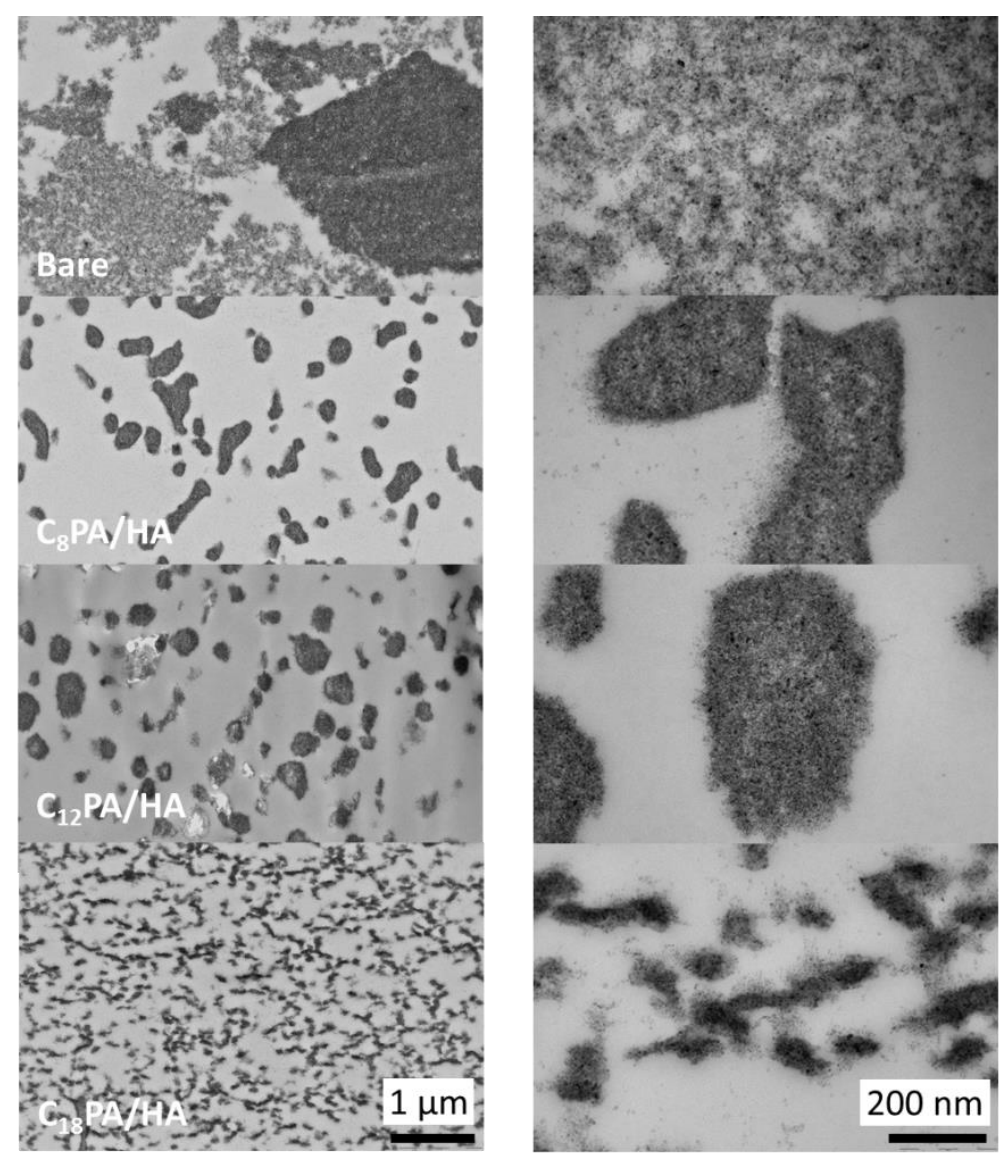

Figure 3: TEM pictures of annealed $\mathrm{TiO}_{2}$-PMMA nanocomposites at nominal volume fraction $\Phi=5 \% \mathrm{v}$, in absence of grafting, and with $\mathrm{C}_{8} \mathrm{PA} / \mathrm{HA}, \mathrm{C}_{12} \mathrm{PA} / \mathrm{HA}$, and $\mathrm{C}_{18} \mathrm{PA} / \mathrm{HA}$, respectively, for two magnifications.

All nanocomposites studied up to here have been formulated with hexylamine to reduce aggregation in chloroform, and annealed at high temperatures, above $\mathrm{T}_{\mathrm{g}}$. The impact of both HA and annealing has been investigated on nanocomposites with $\mathrm{C}_{12} \mathrm{PA}$-grafted NPs, and the result for the structure is shown in Figure 4 for one NP concentration. The intensities are again compared to the form factor of the primary beads. In Figure 4, these intensities can be separated in two subsets of identical structure: those grafted with only $\mathrm{C}_{12} \mathrm{PA}$, and those grafted with a combination of $\mathrm{C}_{12} \mathrm{PA}$ and $\mathrm{HA}$. Annealing the 
nanocomposites $48 \mathrm{~h}$ at $140^{\circ} \mathrm{C}$ thus has not impact on the dispersion of the nanoparticles, the intensity curves for annealed and non-annealed superimposing perfectly for both types of grafting.
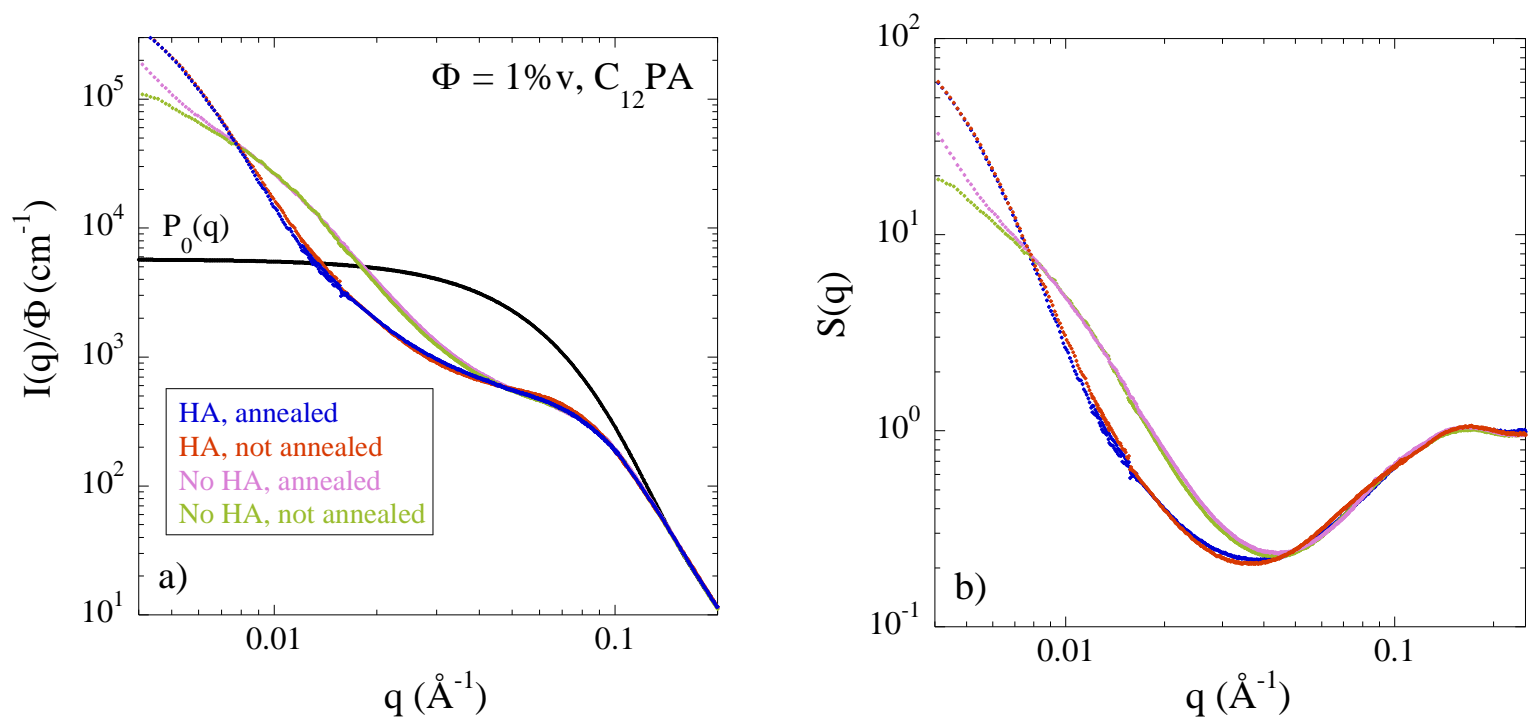

Figure 4: a) Reduced intensity $\mathrm{I}(\mathrm{q}) / \Phi$ of $\mathrm{TiO}_{2}$-PMMA nanocomposites at nominal volume fraction $\Phi=1 \% \mathrm{v}$ grafted with $\mathrm{C}_{12} \mathrm{PA}$, or $\mathrm{C}_{12} \mathrm{PA} / \mathrm{HA}$, annealed or not, compared to the primary particle form factor. b) Corresponding structure factors.

On the other hand, the addition of HA has an impact on the structure at intermediate scales around $\mathrm{q} \approx$ $0.02 \AA^{-1}$, while the high-q shoulder superimposes perfectly for all four samples. As a consequence, the $\mathrm{S}$ (q) plot in Figure $4 \mathrm{~b}$ shows that the interaction peak at high $\mathrm{q}$ is the same for all four systems. Starting from the peak, the structure factors reach a correlation hole of approximately the same depth, which is indicative of similar local densities. We will see below that this is indeed the case, with very close $\kappa$-values. At lower q, the curves separate into different upturns around $0.05 \AA^{-1}$ : in presence of $\mathrm{HA}$, the correlation hole extends down to lower $\mathrm{q}$, indicating a larger size range over which the system is homogeneous. An interpretation in real-space of the scattering shown in Figure 4 might be that locally NPs are organized in zones with the same density or number of neighbors, and that these zones are bigger in presence of HA.

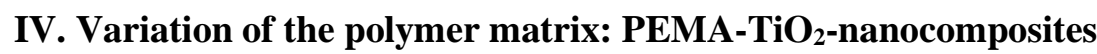

A second set of nanocomposites has been formulated with a different acrylate polymer, PEMA, in order to see if the tendencies induced by surface modification with phosphonic acid molecules are robust with respect to several system changes: first, the additional ethyl group on the monomer makes the polymer slightly more hydrophobic. Secondly, hexylamine was not added; according to Figure 4 its absence leads to slightly smaller aggregates of identical internal density. Thirdly, PEMAnanocomposites have not been annealed, which we have shown in Figure 4 not to affect the local 
structure. We have prepared $\mathrm{TiO}_{2}$-nanocomposites with a single low NP loading (1\%v). In Figure 5a, the scattering of PEMA nanocomposites is shown, and compared to the primary NP form factor $\mathrm{P}_{0}(\mathrm{q})$. The impact of surface modification is very similar to the one obtained in the annealed PMMAnanocomposites with HA shown in Figure 2, with however comparatively higher intensities in the correlation hole: $\mathrm{C}_{18} \mathrm{PA}$ grafting displays the weakest correlation hole (around $\mathrm{q} \approx 0.05 \AA^{-1}$ ), and this hole deepens as the mass of the graft is decreased: smaller grafts lead to denser local assemblies, as in PMMA. Changing the hydrophobicity of the acrylate polymer by increasing the mass of the side group from $\mathrm{CH}_{3}$ to $\mathrm{CH}_{2}-\mathrm{CH}_{3}$ thus does not fundamentally modify the effect of PA-grafting on NPinteractions in terms of the effect of the graft hydrophobicity, but seems to favor less dense aggregates.

In order to prepare the quantitative analysis based on eq.(1), the experimental structure factor has been determined, and the result is shown in Figure $5 \mathrm{~b}$. The minimum of the structure factor $\mathrm{S}_{0}$, i.e., the 'depth' of the correlation hole, is seen to be higher than in Figure 2b, and equally sensitive to the type of graft, as already noticed on the scattered intensity in Figure 5a. The value of the structure factor at the minimum will be interpreted below in terms of local NP volume fractions for all nanocomposites studied in this paper using eq.(1). Finally, one may note as in Figure 2 that the $\mathrm{C}_{18} \mathrm{PA}$-grafts lead to a clearly visible structure at low-q, i.e., a change in slope around $0.007 \AA^{-1}$. This is again the signature of small enough NP assemblies formed in presence of $\mathrm{C}_{18} \mathrm{PA}$. For comparison, the structure factor of the PMMA nanocomposite with HA and the $\mathrm{C}_{18}$-graft is superimposed in Figure $5 \mathrm{~b}$. It can be seen to change slope at lower q, just above the arrow, as compared to the $\mathrm{C}_{18}$-PEMA sample, with a $\mathrm{S}(\mathrm{q})$ bending just below the arrow. This illustrates that HA induces bigger structures.
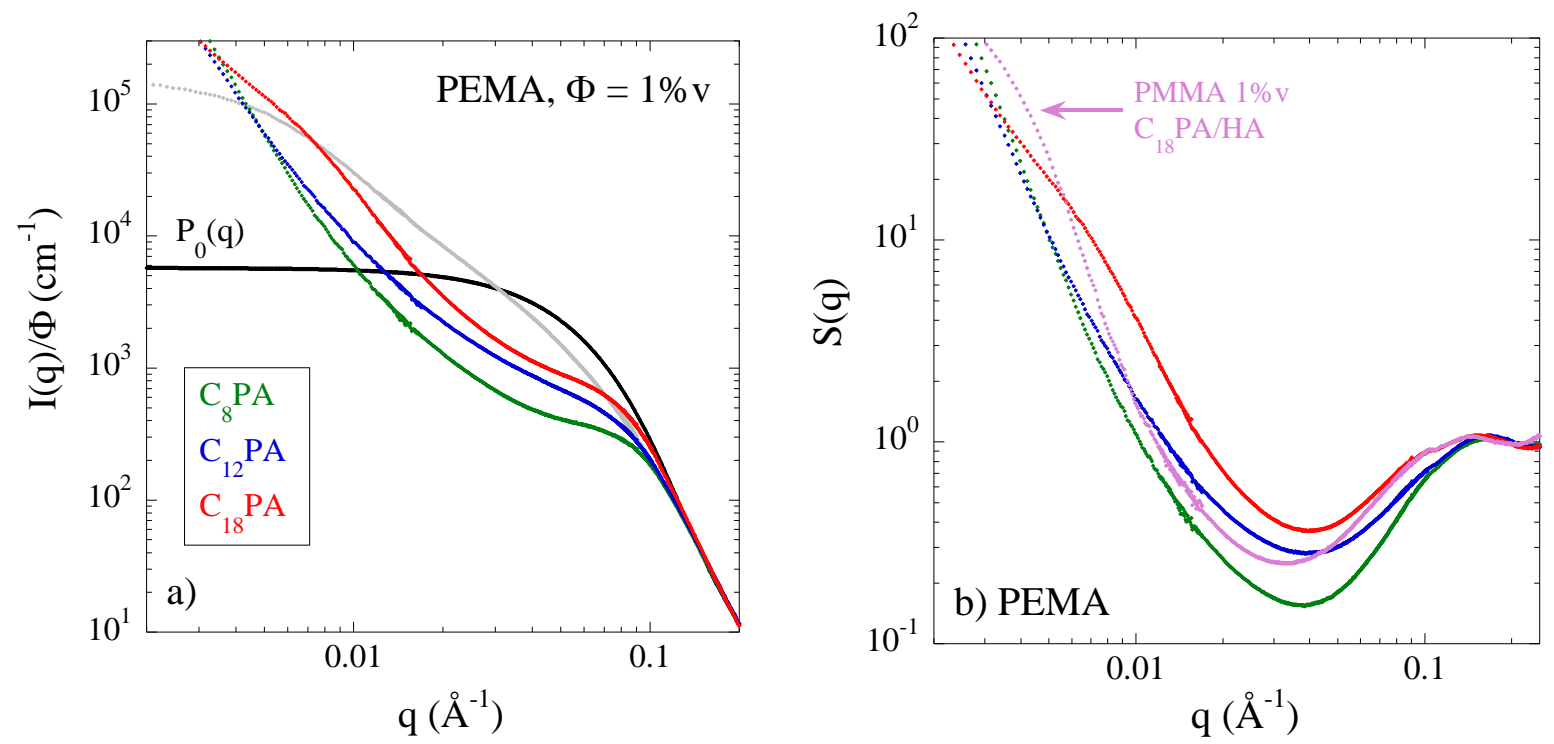

Figure 5: (a) Reduced intensity $\mathrm{I}(\mathrm{q}) / \Phi$ of $\mathrm{TiO}_{2}$-PEMA nanocomposites at nominal volume fraction $\Phi=1 \% \mathrm{v}$, with surface modification with $\mathrm{C}_{8} \mathrm{PA}, \mathrm{C}_{12} \mathrm{PA}$, and $\mathrm{C}_{18} \mathrm{PA}$, respectively, compared to the $\mathrm{TiO}_{2}$ primary particle form factor $\mathrm{P}_{0}(\mathrm{q})$. (b) The apparent structure factor obtained by dividing $\mathrm{I}(\mathrm{q})$ by $\mathrm{P}_{0}(\mathrm{q})$. The PMMA/HA nanocomposite structure factor with $\mathrm{C}_{18} \mathrm{PA}$ (annealed) is reproduced for comparison. 
Before going into the quantitative analysis in the next section, let us have a look at the structure in direct space, as shown by TEM in Figure 6. On the biggest scale shown in the left column, large NP assemblies are again found as with PMMA in Figure 3. With increasing alkyl-chain length of the grafts going downwards in the Figure, the size of the aggregates decreases, as shown at a greater magnification in the right column. Indeed, as suspected from the SAXS data in Figure 5, the surface modification with $\mathrm{C}_{18} \mathrm{PA}$-grafts leads to characteristic cluster sizes around $100 \mathrm{~nm}$. Finally, as with PMMA, the NP clusters appear to be more evenly distributed across the matrix, indicating a higher compatibility with the matrix polymer than the other grafted NPs.
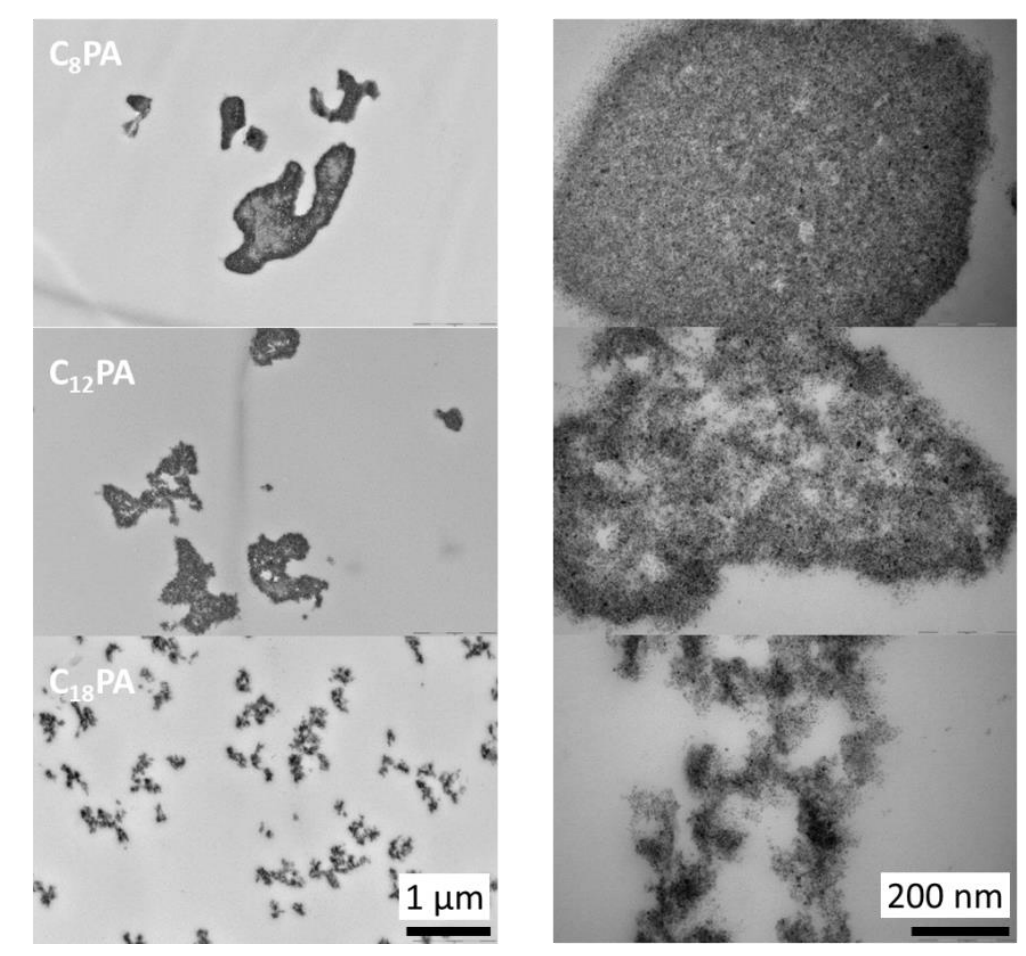

Figure 6: TEM pictures of $\mathrm{TiO}_{2}$-PEMA nanocomposites at nominal volume fraction $\Phi=1 \% \mathrm{v}$ with $\mathrm{C}_{8} \mathrm{PA}$, $\mathrm{C}_{12} \mathrm{PA}$, and $\mathrm{C}_{18} \mathrm{PA}$, respectively, for two magnifications.

\section{Determination of local nanoparticle volume fractions in $\mathrm{TiO}_{2}$-nanocomposites}

We now apply the correlation hole analysis based on eq.(1) to the local structure of $\mathrm{TiO}_{2}-$ polymernanocomposites. The purpose of our mapping procedure is to deduce the aggregate compacity from the experimentally determined 'depth' of the correlation hole. The experimental structure factors as the ones shown in Figures $2 b, 4 b$ and $5 b$ have been determined for all nanocomposite intensities. $S_{0}$ is taken as the lowest value of $\mathrm{S}(\mathrm{q})$, and it is entered in eq.(1) to determine $\kappa$, using $\alpha=0.59$. The results for PMMA- and PEMA-nanocomposites are shown as a function of nanoparticle volume fraction and grafting in Figure 7a and 7b, respectively. 

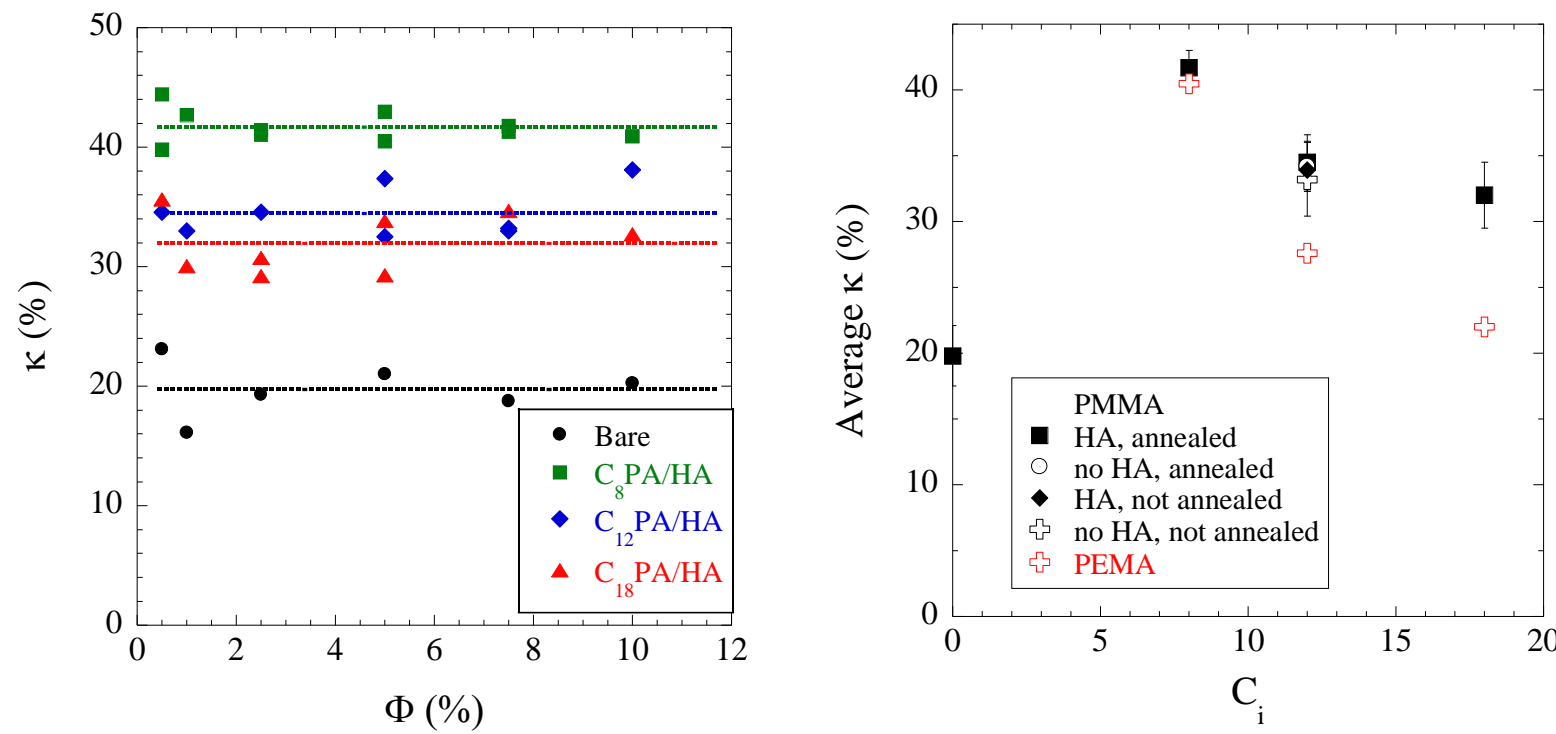

Figure 7: Local NP volume fractions $\kappa$ deduced from the depth of the correlation hole of the structure factors of (a) annealed HA-PMMA-nanocomposites as a function of nominal volume fraction $\Phi$, for bare NPs (circles), and NPs grafted with $\mathrm{C}_{8} \mathrm{PA}$ (squares), $\mathrm{C}_{12} \mathrm{PA}$ (diamonds), and $\mathrm{C}_{18} \mathrm{PA}$ (triangles), respectively. (b) $\kappa$ averaged over the volume fraction as shown by the horizontal line in Figure a, as a function of the number of carbon atoms in the grafts. The red crosses are the results for PEMA, all others for PMMA.

A first striking result of Figure 7a was already observed in the original intensities in Figure 1: the depth of the correlation hole and thus the local particle densities are independent of the particle volume fraction, and the corresponding $\mathrm{\kappa}$ do not vary with $\Phi$, as shown by the horizontal lines representing the averages. This is different from what one might expect, as higher particle concentrations may be seen as a thermodynamic driving force towards denser local assemblies. Presumably due to the novelty of the present correlation hole analysis, such data are usually not available in the literature for comparison. Using this analysis, we have recently characterized a model system, with well-defined phosphonic acid-modified nanoparticles in suspension. ${ }^{26}$ Nanocomposites have been produced via a latex-route, and the compacity was found to increase with the global volume fraction for all types of grafts. ${ }^{27}$ On the other hand, we have recently shown that in simplified industrial nanocomposites made with industrial (polydisperse) silica filler following a mechanical mixing route also have rather constant (albeit higher) local particle volume fractions. ${ }^{14}$ In conclusion, both tendencies of $\kappa$ with $\Phi$ seem to exist.

The next result evidenced by Figure $7 \mathrm{~b}$ is that the depth of the correlation hole remains independent of the total volume fraction, but changes with the type of the PA graft, as already observed in Figure 2 for PMMA-, and Figure 5 for PEMA-nanocomposites. For the latter, the aggregate compacity $\kappa$ is found to decrease by about a factor of two, from some $40 \%$ to about $20 \%$, as the length of the graft reported on the $\mathrm{x}$-axis is increased: the system tends towards a better dispersion. The same is true with the PMMA-nanocomposites, the compacities of which are also reported in Figure $7 \mathrm{~b}$. They decrease, but seem to level off at around $30 \%$. This result represents the quantitative evidence that the more 
hydrophobic matrix, PEMA, yields nanocomposites that are more sensitive to the NP surface modification, and in particular to modifications with the most hydrophobic graft C18PA. It is supposed that the different chain mass does not influence the compacity, due to the solvatation of the chains assuring a high-chain mobility, favoring equilibration of the system, and thus dominant enthalpic contributions.

It is not easy to conclude that this the observed evolution from PMMA to PEMA is consistent with visual inspection of the TEM micrographs shown in Figure 3 and 6, as the scattering analysis is much more quantitative. However, the dispersion in nanocomposites does seem to improve from $\mathrm{C}_{8} \mathrm{PA}$ to $\mathrm{C}_{18} \mathrm{PA}$. Concerning the bare nanoparticles, one must keep in mind that they have been formulated using a completely different procedure, as phase transfer does not take place without PAs. The low compacity of these samples may be due to the result of the input of mechanical energy used to disperse these NPs. One should also keep in mind that the function given in eq.(1) is convex, i.e. it possesses an upward curvature. When applying it to systems presenting domains of low and high densities, then two different correlation holes will be averaged. Due to the curvature of the function in eq.(1), this averaging of $\mathrm{S}(\mathrm{q})$ leads to a compacity which is lower than the weighted average of $\kappa$. In other words, applying eq.(1) to heterogeneous samples like the bare-NP nanocomposites might provide underestimations of $\kappa$, which explains its possibly too low value. Moreover, the absence of phosphonic acids allows for strong interactions between metal oxide surfaces and acrylates, which adds a different contribution to the thermodynamics of the system ${ }^{37}$.

As a last point, the $\mathrm{C}_{12}$ PA-PMMA nanocomposite has also been used to explore the influence of annealing and HA addition, cf. Figure 4. The corresponding data points have also been determined from an average procedure over all $\Phi$, and they are superimposed in Figure 7b. Clearly, all four samples (HA/annealing) have an identical local volume fraction of $\kappa=34 \%$, within error bars (2\%), and it may be concluded that neither HA nor annealing influence the local particle concentrations.

\section{Conclusion}

The influence of surface-modification of $\mathrm{TiO}_{2}$-nanoparticles on the density of their assemblies in polymer nanocomposites has been studied by SAXS and TEM, using a newly developed correlationhole analysis. The compacity к, i.e. local volume fraction of NPs, was found to be independent of the global particle volume fraction in the range of study, up to $\Phi=10 \% \mathrm{v}$. NPs grafted with short-chain PAs $\left(\mathrm{C}_{8} \mathrm{PA}\right)$ arrange rather densely $(\kappa \approx 40 \%)$ in the polymer matrix. Grafting longer chains $\mathrm{C}_{\mathrm{n}} \mathrm{PA}$ induces a monotoneous decrease of $\mathrm{K}$ with increasing the chain length, indicating a better compatibility of the surface-modified NPs with the polymer. The effect is similar but stronger for samples produced with a more hydrophobic polymer matrix, PEMA. 
The influence of two other parameters has also been investigated, hexylamine and annealing. HA is sometimes added to stabilize NPs in suspension. The PMMA-series was produced with both, whereas the PEMA-series had neither. The influence of each parameter separately has been tested on PMMAnanocomposites with $\mathrm{C}_{12} \mathrm{PA}$-grafting. It was found that annealing had virtually no effect on the dispersion, whereas HA led to larger NP assemblies of the same density.

In conclusion, we have shown that scattered intensities of original nanocomposite systems can be interpreted quantitatively in the intermediate q-range, using a simple numerical relationship between the height of the structure factor at the correlation hole and the local volume fraction $\kappa$. The quantitative analysis of the correlation hole is thus established as an appropriate tool of structural analysis of nanoparticle assemblies, and it is hoped that this approach can be generalized easily to many other systems.

Acknowlegements: The authors are thankful for beamtime on beamline Swing by Synchrotron Soleil (St Aubin, France). The Ph.D. of C.S.P. was financed by Labex ChemiSyst under contract number ANR-10-LABX-05-01. Support by the ANR NANODYN project, grant ANR-14-CE22-0001-01 of the French Agence Nationale de la Recherche, is also acknowledged. We are indebted to Lotus Synthesis for the gift of NPs. Access to TEM measurements at the University of Montpellier and support by F. Godiard and V. Richard are acknowledged.

Supporting Information. Additional scattering data for PMMA nanocomposites. Large-scale TEM pictures and additional pictures for $2.5 \% \mathrm{v}$-PMMA nanocomposites.

\section{References}

(1) Mark, J. E.; Erman, B.; Eirich, F. R., Science and Technology of Rubber. Academic Press: San Diego, 1994.

(2) Oberdisse, J. Structure and rheological properties of latex-silica nanocomposite films: Stressstrain isotherms. Macromolecules 2002, 35 (25), 9441-9450.

(3) Robertson, C. G.; Roland, C. M. Glass Transition and Interfacial Segmental Dynamics in Polymer-Particle Composites. Rubber Chem. Technol. 2008, 81 (3), 506-522. 
(4) Jancar, J.; Douglas, J. F.; Starr, F. W.; Kumar, S. K.; Cassagnau, P.; Lesser, A. J.; Sternstein, S. S.; Buehler, M. J. Current issues in research on structure-property relationships in polymer nanocomposites. Polymer 2010, 51 (15), 3321-3343.

(5) Schmidt, G.; Malwitz, M. M. Properties of polymer-nanoparticle composites. Curr. Opin. Colloid Interface Sci 2003, 8 (1), 103-108.

(6) Baeza, G. P.; Oberdisse, J.; Alegria, A.; Couty, M.; Genix, A. C. A high-temperature dielectric process as a probe of large-scale silica filler structure in simplified industrial nanocomposites. Phys. Chem. Chem. Phys. 2015, 17, 1660-1666.

(7) Baeza, G. P.; Oberdisse, J.; Alegria, A.; Saalwaechter, K.; Couty, M.; Genix, A.-C. Depercolation of aggregates upon polymer grafting in simplified industrial nanocomposites studied with dielectric spectroscopy. Polymer 2015, 73, 131-138.

(8) Boonstra, B. B. Role of particulate fillers in elastomer reinforcement: a review. Polymer 1979, 20 (6), 691-704.

(9) Heinrich, G.; Kluppel, M.; Vilgis, T. A. Reinforcement of elastomers. Curr. Opin. Solid State Mater. Sci. 2002, 6 (3), 195-203.

(10) Kohls, D. J.; Beaucage, G. Rational design of reinforced rubber. Curr. Opin. Solid State Mater. Sci. 2002, 6 (3), 183-194.

(11) Tatou, M.; Genix, A. C.; Imaz, A.; Forcada, J.; Banc, A.; Schweins, R.; Grillo, I.; Oberdisse, J. Reinforcement and polymer mobility in silica-latex nanocomposites with controlled aggregation. Macromolecules 2011, 44, 9029-9039.

(12) Banc, A.; Genix, A. C.; Chirat, M.; Dupas, C.; Caillol, S.; Sztucki, M.; Oberdisse, J. Tuning structure and rheology of silica-latex nanocomposites with the molecular weight of matrix chains: a coupled SAXS-TEM-simulation approach. Macromolecules 2014, 47 (9), 3219-3230.

(13) Janes, D. W.; Moll, J. F.; Harton, S. E.; Durning, C. J. Dispersion Morphology of Poly(methyl acrylate)/Silica Nanocomposites. Macromolecules 2011, 44 (12), 4920-4927.

(14) Baeza, G. P.; Genix, A. C.; Degrandcourt, C.; Petitjean, L.; Gummel, J.; Couty, M.; Oberdisse, J. Multiscale Filler Structure in Simplified Industrial Nanocomposite Silica/SBR Systems Studied by SAXS and TEM. Macromolecules 2013, 46 (1), 317-329.

(15) Baeza, G. P.; Genix, A. C.; Degrandcourt, C.; Gummel, J.; Couty, M.; Oberdisse, J. Mechanism of aggregate formation in simplified industrial silica styrene-butadiene nanocomposites: effect of chain mass and grafting on rheology and structure. Soft Matter 2014, 10, 6686-6695.

(16) Baeza, G. P.; Genix, A. C.; Degrandcourt, C.; Petitjean, L.; Gummel, J.; Schweins, R.; Couty, M.; Oberdisse, J. Effect of Grafting on Rheology and Structure of a Simplified Industrial Nanocomposite Silica/SBR. Macromolecules 2013, 46 (16), 6388-6394.

(17) Baeza, G. P.; Genix, A. C.; Degrandcourt, C.; Gummel, J.; Mujtaba, A.; Saalwächter, K.; Thurn-Albrecht, T.; Couty, M.; Oberdisse, J. Studying twin-samples provides evidence for a 
unique structure-determining parameter in simplified industrial nanocomposites. ACS Macro Lett. 2014, 3, 448-452.

(18) Kumar, S. K.; Jouault, N.; Benicewicz, B.; Neely, T. Nanocomposites with Polymer Grafted Nanoparticles. Macromolecules 2013, 46 (9), 3199-3214.

(19) Chevigny, C.; Dalmas, F.; Di Cola, E.; Gigmes, D.; Bertin, D.; Boue, F.; Jestin, J. PolymerGrafted-Nanoparticles Nanocomposites: Dispersion, Grafted Chain Conformation, and Rheological Behavior. Macromolecules 2011, 44 (1), 122-133.

(20) Bouty, A.; Petitjean, L.; Degrandcourt, C.; Gummel, J.; Kwaśniewski, P.; Meneau, F.; Boué, F.; Couty, M.; Jestin, J. Nanofiller Structure and Reinforcement in Model Silica/Rubber Composites: A Quantitative Correlation Driven by Interfacial Agents. Macromolecules 2014, 47 (15), 5365-5378.

(21) Musino, D.; Genix, A. C.; Fayolle, C.; Papon, A.; Guy, L.; Meissner, N.; Weda, P.; Kozak, R.; Bizien, T.; Chaussée, T.; Oberdisse, J. Synergistic effect of small molecules on large-scale structure of simplified industrial nanocomposites. Macromolecules 2017, in press.

(22) Stockelhuber, K. W.; Svistkov, A. S.; Pelevin, A. G.; Heinrich, G. Impact of Filler Surface Modification on Large Scale Mechanics of Styrene Butadiene/Silica Rubber Composites. Macromolecules 2011, 44 (11), 4366-4381.

(23) Meth, J. S.; Zane, S. G.; Chi, C.; Londono, J. D.; Wood, B. A.; Cotts, P.; Keating, M.; Guise, W.; Weigand, S. Development of Filler Structure in Colloidal Silica-Polymer Nanocomposites. Macromolecules 2011, 44 (20), 8301-8313.

(24) Zhang, Q.; Archer, L. A. Poly(ethylene oxide)/silica nanocomposites: Structure and rheology. Langmuir 2002, 18 (26), 10435-10442.

(25) Lin, Y.; Liu, L.; Zhang, D.; Liu, Y.; Guan, A.; Wu, G. Unexpected segmental dynamics in polystyrene-grafted silica nanocomposites. Soft Matter 2016, 12 (41), 8542-8553.

(26) Schmitt Pauly, C.; Genix, A.-C.; Alauzun, J. G.; Sztucki, M.; Oberdisse, J.; Mutin, P. H. Surface modification of alumina-coated silica nanoparticles in aqueous sols with phosphonic acids and impact on nanoparticle interactions. Phys. Chem. Chem. Phys. 2015, 17 (29), 1917319182.

(27) Schmitt Pauly, C.; Genix, A.-C.; Alauzun, J. G.; Jestin, J.; Sztucki, M.; Mutin, P. H.; Oberdisse, J. Structure of alumina-silica nanoparticles grafted with alkylphosphonic acids in poly(ethylacrylate) nanocomposites. Polymer 2016, 97, 138-146.

(28) Pauly, C. S.; Genix, A. C.; Alauzun, J. G.; Guerrero, G.; Appavou, M. S.; Perez, J.; Oberdisse, J.; Mutin, P. H. Simultaneous Phase Transfer and Surface Modification of TiO2 Nanoparticles Using Alkylphosphonic Acids: Optimization and Structure of the Organosols. Langmuir 2015, 31 (40), 10966-10974.

(29) Genix, A. C.; Oberdisse, J. Estimating the local density of nanoparticle assemblies. Submitted. 
(30) Nakayama, N.; Hayashi, T. Preparation of TiO2 nanoparticles surface-modified by both carboxylic acid and amine: Dispersibility and stabilization in organic solvents. Colloids Surf., A 2008, $317(1-3), 543-550$.

(31) Nakayama, N.; Hayashi, T. Preparation and characterization of poly(l-lactic acid)/TiO2 nanoparticle nanocomposite films with high transparency and efficient photodegradability. Polym. Degrad. Stabil. 2007, 92 (7), 1255-1264.

(32) Percus, J. K.; Yevick, G. J. Analysis of Classical Statistical Mechanics by Means of Collective Coordinates. Phys Rev 1958, 110 (1), 1-13.

(33) Ashcroft, N. W.; Langreth, D. C. Structure of Binary Liquid Mixtures. I. Phys Rev 1967, 156 (3), 685-692.

(34) Hansen, J. P.; McDonald, I. R., Theory of Simple Liquids. Academic Press: London, 1986.

(35) Carnahan, N. F.; Starling, K. E. Equation of State for Nonattracting Rigid Spheres. J. Chem. Phys. 1969, 51 (2), 635-636.

(36) Lindner, P., Neutrons, X-ray and Light Scattering. North Holland, Elsevier: 2002.

(37) Papon, A.; Saalwächter, K.; Schäler, K.; Guy, L.; Lequeux, F.; Montes, H. Low-Field NMR Investigations of Nanocomposites: Polymer Dynamics and Network Effects. Macromolecules 2011, 44 (4), 913-922. 


\section{FOR TABLE OF CONTENTS USE ONLY}

Tuning of local nanoparticle arrangements in $\mathrm{TiO}_{2}$-polymer nanocomposites by grafting of phosphonic acids

Anne-Caroline Genix, Céline Schmitt-Pauly, Johan G. Alauzun, Thomas Bizien, P. Hubert Mutin, Julian Oberdisse

$\mathrm{TiO}_{2}$-PMMA nanocomposites

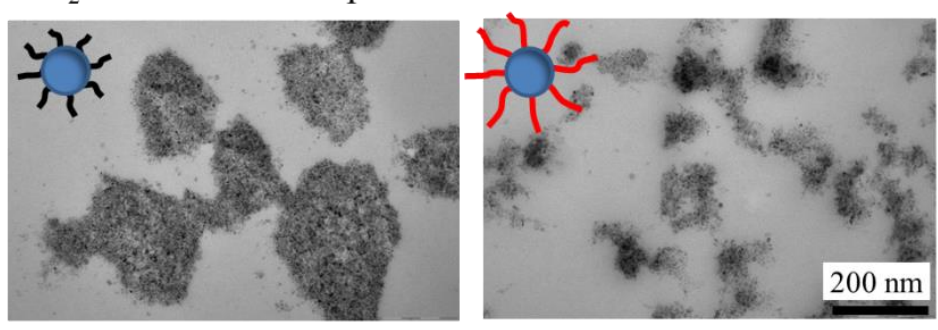

compatibilization 